\title{
Hosoya polynomial and topological indices of $n$-linear benzene
}

\author{
Abdul Rauf Nizami ${ }^{*}$, Muhammad Idrees ${ }^{2}$, Numan Amin ${ }^{3}$ and Zaffar Iqbal ${ }^{4}$ \\ ${ }^{I}$ Faculty of Information Technology, University of Central Punjab, Lahore, Pakistan. \\ ${ }^{2}$ Division of Science and Technology, University of Education, Lahore, Pakistan. \\ ${ }^{3}$ Abdus Salam School of Mathematical Sciences, GC University, Lahore, Pakistan. \\ ${ }^{4}$ Department of Mathematics, University of Gujrat, Pakistan.
}

\begin{abstract}
The Hosoya polynomial was introduced by Hosoya in 1988 for a molecular graph $G$ as $H(G, x)=\sum_{k=1}^{d(G)} d(G, k) x^{k}$, where $d(G, k)$ is the number of pairs of vertices of $G$ laying at distance $k$ from each other, to count the number of paths of different lengths in $G$. The most interesting application of the Hosoya polynomial is that almost all distance-based topological indices can be recovered from it. In this article, we give the general closed form of the Hosoya polynomial of $n$ times linearly concatenated benzene molecule $B_{n}$ by partitioning the vertices of $B_{n}$ and by using induction to find sums of distances of different lengths in $B_{n}$. We also find Wiener, hyper Wiener, Harary, and TSZ indices to predict physical, chemical and pharmacological properties of molecules containing $B_{n}$.
\end{abstract}

Keywords: Hosoya polynomial, topological indices, linear benzene.

\section{INTRODUCTION}

The Hosoya polynomial of a graph was introduced by $\mathrm{H}$. Hosoya in 1988 as a counting polynomial, which counts the number of distances of paths of different lengths in the graph (Hosoya, 1988).

In 1993, Gutman introduced Hosoya polynomial for a vertex of a graph (Gutman, 1993), which is related to Hosoya polynomial of the graph. The most significant application of the Hosoya polynomial is that almost all distance-based graph invariants, which are used to predict physical, chemical and pharmacological properties of organic molecules, can be recovered from it.
Hosoya polynomial has been computed for several classes of graphs. In 2002, Diudea computed the Hosoya polynomial of several classes of toroidal nets and recovered their Wiener indices (Diudea, 2002). In 2011, Ali and Ahmed found the Hosoya polynomial of concatenated pentagonal rings (Ali \& Ahmed, 2011). In 2012, Narayankar et al. gave a recursive method for calculating the Hosoya polynomial of Hanoi graphs, and computed some of their distance-based invariants (Narayankar et al., 2012). In 2013, Farahani computed the Hosoya polynomial of polycyclic aromatic hydrocarbons (Farahani, 2013).

There are some useful topological indices that are related to Hosoya polynomial such as Wiener, hyper Wiener, Tratch-Stankevitch-Ziefirov (TSZ), and Harary indices. The Wiener index was introduced by Harry Wiener in 1947 and was used to correlate with boiling points of alkanes (Wiener, 1947). Later, it was observed that the Wiener index can be used to determine a number of physico-chemical properties of alkanes such as heat of formation, heat of vapourization, molar volumes and molar refractions (Gutman \& Polansky, 1986). Moreover, it can be used to correlate those physicochemical properties which depend on the volume-surface ratio of molecules and to gas-chromatographic retention data for a series of structurally related molecules. The hyper-Wiener introduced by Randic in 1993 is another topological index and most of the mathematical properties of it were investigated. It is also used to predict physico-

*Corresponding author (arnizami@ucp.edu.pk; (10) https://orcid.org/0000-0003-3624-6160) 
chemical properties of organic compounds, particularly pharmacology, agriculture, and environment protection (Cash, 2002); for more details, see also (Gutman, 1993; Randic, 1993; Lukovits, 1994; Klein, 1995; Diudea, 1996). In 1993, Plavsic et al. introduced a new topological index, known as Harary index, to characterise chemical graphs (Plavsic et al., 1993). In 1990, Tratch, Stankevitch and Zefirov introduced Tratch-StankevitchZefirov (TSZ) index, which was obtained by expanding the Wiener index.

This article provides information on the general form of the Hosoya polynomial of the graph that corresponds to linearly concatenated benzene molecules and its Wiener, hyper Wiener, TSZ and Harary indices.

\section{METHODOLOGY}

Some basic notations and definitions, which are required for the rest of the article, are included in this section.

A graph $G$ is a pair $(V, E)$, where $V$ is the set of vertices and $E$ is the set of edges. A path from a vertex $v$ to a vertex $w$ in a graph is a sequence of vertices and edges that starts from $v$ and stops at $w$. The number of edges in a path is the length of that path. A graph is said to be connected if there is a path between any two of its vertices. The distance $d(u, v)$ between two vertices $u, v$ of a connected graph $G$ is the length of a shortest path between them. The diameter of $G$, denoted by $d(G)$, is the longest distance in $G$.

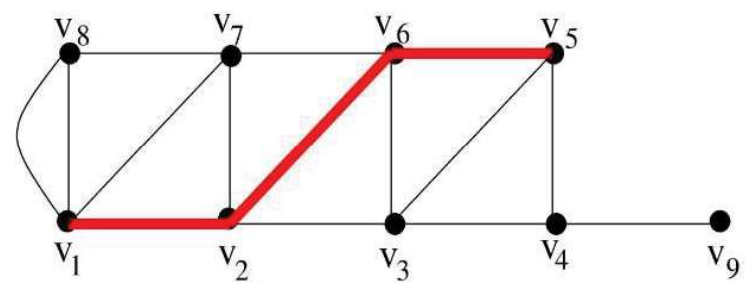

Figure 1: A connected graph with a highlighted shortest path between two vertices

A molecular graph is a representation of the structural formula of a chemical compound in terms of graph theory. Specifically, molecular graph is a graph with vertices corresponding to atoms of the compound and edges corresponding to chemical bonds; however, hydrogen atoms are often omitted.
Definition 1. The Hosoya polynomial in a variable $x$ of a molecular graph $G=(V, E)$ is defined as

$H(G, x)=\sum_{\{v, u\} \in V} x^{d(u, v)}=\sum_{k=1}^{d(G)} d(G, k) x^{k}$

where $d(G, k)$ is the number of pairs of vertices of $G$ laying at distance $k$ from each other.

A function $I$ which assigns a unique number $I(G)$ in connected graph $G$ is called a graph invariant. Instead of the function $I$, it is custom to write the number $I(G)$ as the graph invariant. An invariant of a molecular graph is called a topological index if it is used to determine structure-property or structure-activity correlation.

In the following, $d(u, v)$ is the distance between two arbitrary vertices $u, v$ of $G$ and $d(v, G)$ is the sum of distances of $v$ with all vertices of a connected graph $G$.

Definition 2. The Wiener index $W(G)$ of a graph $G$ is defined as

$W(G)=\sum_{v<u} d(v, u)=\frac{1}{2} \sum_{v \in V} d(v, G)$

The Wiener index and the Hosoya polynomial are related by the equation

$W(G)=\left.\frac{d}{d x} H(G, x)\right|_{x=1}$

Definition 3. The hyper-Wiener index $W W(G)$ of graph $G$ is defined as

$W W(G)=\sum_{v<u} d(v, u)=\frac{1}{2} \sum d(v, u)^{2}+\frac{1}{2} \sum d(v, u)$

The hyper-Wiener index and the Hosoya polynomial are related by the equation

$$
W W(G)=\left.\frac{1}{2} \frac{d^{2}}{d x^{2}} x H(G, x)\right|_{x=1}
$$

Definition 4. The Harary index $H a(G)$ of graph $G$ is defined as

$$
H a(G)=\sum_{i<j} \frac{1}{d\left(u_{i}, v_{j}\right)^{2}} .
$$

The Harary index and the Hosoya polynomial are related by the equation

$$
H a(G)=\int_{0}^{1} \frac{H(G, x)}{x} d x
$$


The Tratch-Stankevitch-Zefirov index is also related to the Hosoya polynomial under the relation

$$
\operatorname{TSZ}(G)=\left.\frac{1}{3 !} \frac{d^{3}}{d x^{3}} x^{2} H(G, x)\right|_{x=1}
$$

The problem was solved directly using the definition of the Hosoya polynomial. The vertices of the graph were partitioned to indicate the paths of different lengths in the graph and induction was used to give the general form of sums of distances of different lengths.

\section{RESULTS AND DISCUSSION}

In this section, the Hosoya polynomial of the graph corresponding to $n$ times concatenated benzene molecule $C_{6} H_{6}$ is given. This graph is denoted by $B_{n}$ and is given in Figure 2.

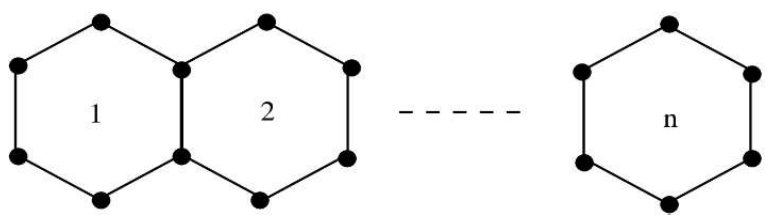

Figure 2: n-Linear benzene, $B_{n}$

In the following $L_{n}$ denotes the matrix that represents the distances among vertices of $B_{n}$

Theorem 1. The Hosoya polynomial of $B_{n}$ is $H\left(B_{n}\right)=\sum_{i=1}^{2 n+1} c_{i} x^{i}$, where

$c_{i}=\left\{\begin{aligned} 5 n+1, & i=1 \\ 9 n-6, & i=3 \\ 8 n-4 i+6, & 2 \leq i \leq 2 n+1, i \neq 3\end{aligned}\right.$

Proof. Depending on $i$ the proof is divided into three parts:

Part I. ( $i=1)$ The number of paths of length one, $c_{1}$, is the total number of edges in the graph, which in this case is $5 n+1$.

Part II. $(i=3)$ Let $D_{i}$ denote the set of vertices that gives $i$ times a path of length 3; this means that each element of $D_{i}$ appears in $i$ distinct paths of length 3 . For $n=1$ $D_{1}=\{1,2,3\}$, thereby $c_{3}=\left|D_{1}\right|=3$. (See Figure 3 and $\left.L_{1}\right)$.
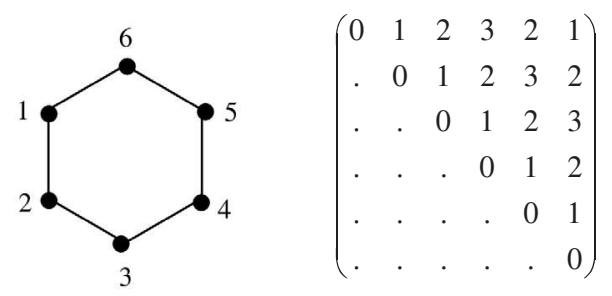

Figure 3: $\quad B$

Matrix $L_{1}$

For $n=2, D_{1}=\{4,6,7\}, D_{2}=\{1,2,5\}$, and $D_{3}=\{3\}$. Thereby $c_{3}=1\left|D_{1}\right|+2\left|D_{2}\right|+3\left|D_{3}\right|=12$. (See Figure 4 and $L_{2}$ )

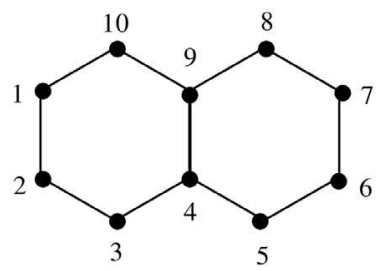

Figure 4: $B_{2}$

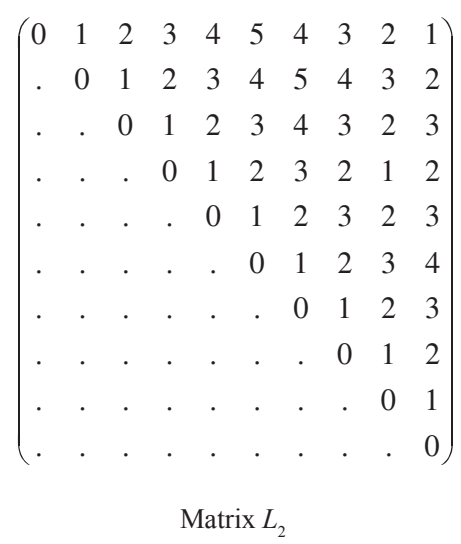

For $n=3, D_{1}=\{8,9,10,11\}, D_{2}=\{1,2,4,6,7\}, D_{3}=\{3\}$, and $D_{4}=\{5\}$. Thereby $c_{3}=1\left|D_{1}\right|+2\left|D_{2}\right|+3\left|D_{3}\right|$ $+4\left|D_{4}\right|=21$. (See Figure 5 and Matrix $L_{3}$ )

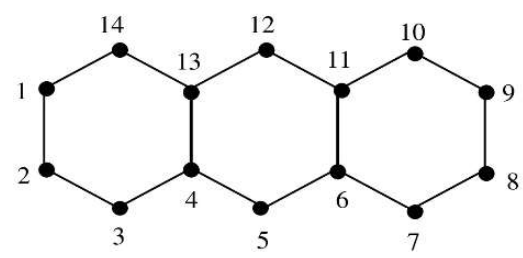

Figure 5: $B_{3}$ 


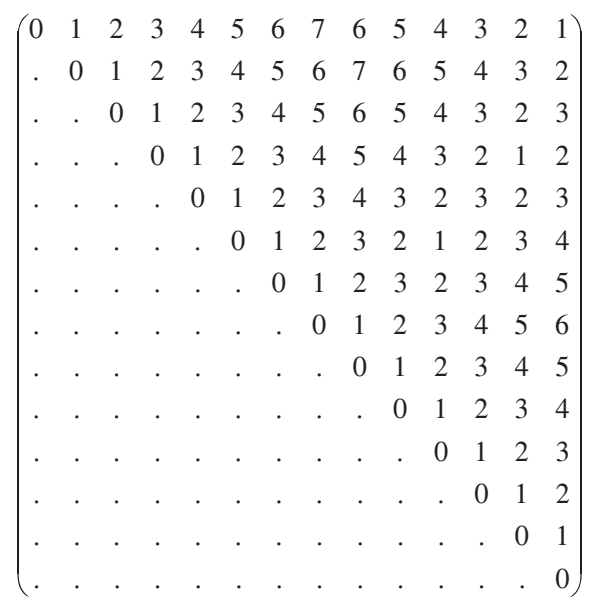

Matrix $L_{5}$

For $\quad n \geq 4, \quad D_{1}=\{2 n+2,2 n+3, \ldots, 4 n-1\}$, $D_{2}=\{1,2,4,2 n, 2 n+1\}, D_{3}=\{3\} \cup\{6,8, \ldots, 2 n-2\}$, and $D_{4}=\{5,7,9, \ldots, 2 n-1\}$. Thereby $c_{3}=1\left|D_{1}\right|+2\left|D_{2}\right|+$ $3\left|D_{3}\right|+4\left|D_{4}\right|=1(2 n-2)+2(5)+3(n-2)+4(n-2)$ $=9 n-6$.

Part III. $(2 \leq i \leq 2 n+1, i \neq 3)$ The authors proved it by induction on $n$ that, by addition of one molecule, the number of paths of length $i$ increases by 8 . For $n=1$ all paths of length 2 are $1 \rightarrow 2 \rightarrow 3,2 \rightarrow 3 \rightarrow 4,3 \rightarrow 4 \rightarrow 5$, $4 \rightarrow 5 \rightarrow 6,5 \rightarrow 6 \rightarrow 1$, and $6 \rightarrow 1 \rightarrow 2$, and one can see 6 bold entries, each of value 2 , in the following distance matrix;

$$
\left(\begin{array}{cccccc}
0 & 1 & \mathbf{2} & 3 & \mathbf{2} & 1 \\
\cdot & 0 & 1 & \mathbf{2} & 3 & \mathbf{2} \\
\cdot & \cdot & 0 & 1 & \mathbf{2} & 3 \\
\cdot & \cdot & . & 0 & 1 & \mathbf{2} \\
\cdot & \cdot & \cdot & . & 0 & 1 \\
\cdot & \cdot & \cdot & . & . & 0
\end{array}\right)
$$

Suppose, the result is true for $n=k, k \geq 4$. That is the number of paths of length $i$ is $8 k-4 i+6$.

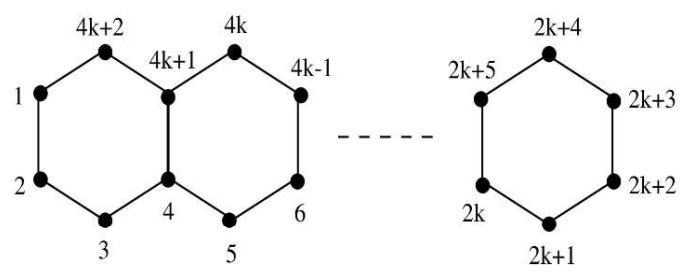

Figure 6: $\quad B_{\mathrm{k}}$ with labels
Note that the addition of one new molecule to $B_{k}$ adds 4 new vertices. These additional vertices are labelled as $a$, $b, c$ and $d$ (Figure 7).

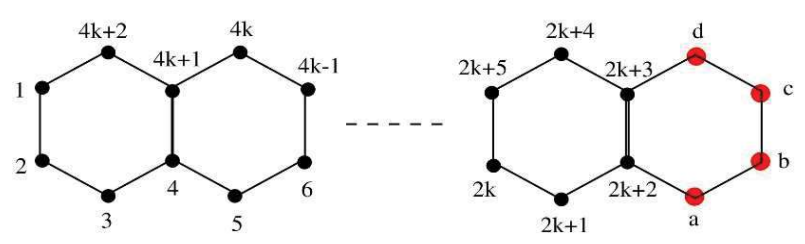

Figure 7: $B_{\mathrm{k}+1}$ with labels

When counting the additional paths, the vertex $a$ contributes 3 distinct paths of length $2: 2 k+1 \rightarrow 2 k+2 \rightarrow a, a \rightarrow b \rightarrow c$, and $2 k+3 \rightarrow$ $2 k+2 \rightarrow a$. Similarly, the vertices $b, c$ and $d$ contribute respectively 2,1 , and 2 paths of length 2 . This adds 8 additional length 2 paths to the number of length 2 paths in the chain of $\mathrm{k}$ molecules. Therefore, the number of length 2 paths in the chain of $k+1$ molecules, $c_{2}$, equals to the summation of number of length 2 paths in the chain of $\mathrm{k}$ and 8. Each of vertex $a, b, c$, and $d$ contributes exactly 2 paths of length $i \geq 4$. These paths for vertex $a$ are $2 k+3-i \rightarrow a$ and $a \rightarrow$ $2 k+2 \rightarrow 2 k+3 \rightarrow \cdots \rightarrow 2 k+1-i$.(Figure 7).

For vertex $b$, the paths are $2 k+4-i \rightarrow b$ and $b \rightarrow c \rightarrow d \rightarrow \cdots \rightarrow 2 k+i$. For vertex $c$, the paths are $2 k+5-i \rightarrow c$ and $c \rightarrow d \rightarrow 2 k+3 \rightarrow 2 k+i+1$ and for $d$ they are $2 k+4-i \rightarrow \cdots \rightarrow 2 k+2 \rightarrow 2 k+3 \rightarrow d$, and $\quad d \rightarrow 2 k+3 \rightarrow 2 k+4 \rightarrow \cdots \rightarrow 2 k+i+2$. This completes the proof.

The above result is demonstrated in the following example for $B_{5}$

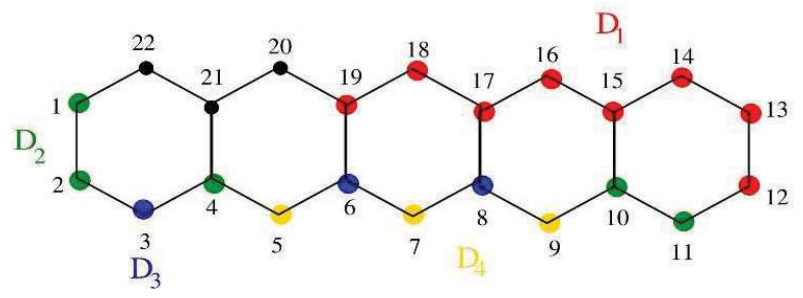

Figure 8: $B_{5}$ with labels

The $22 \times 22$ distance matrix $L_{5}$ corresponding to $B_{5}$ is: 


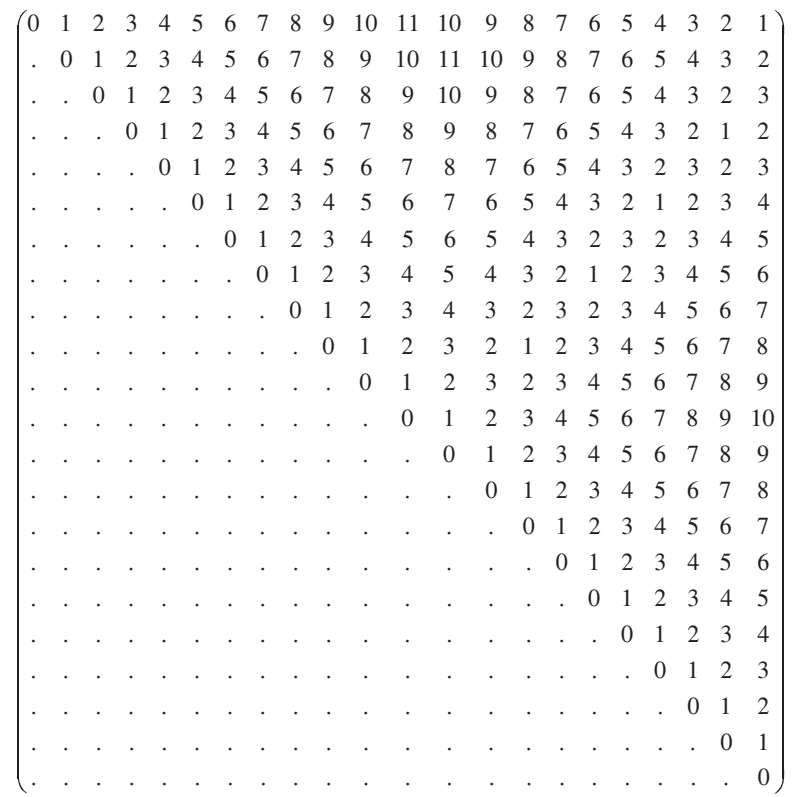

Matrix $L_{5}$

Part I. $(i=1) c_{1}=26$, because the number of edges in $B_{5}$ is 26 ; see Figure 8.

Part II. $(i=3)$ In this case, the vertices are divided into 4 disjoint sets: $D_{1}=\{12,13,14,15,16,17,18,19\}, \quad D_{2}=$
$\{1,2,4,10,11\}, D_{3}=\{3,6,8\}$ and $D_{4}=\{5,7,9\}$. Therefore, $c_{3}=1\left|D_{1}\right|+2\left|D_{2}\right|+3\left|D_{3}\right|+4\left|D_{4}\right|=8+2(5)+3(3)+4(3)$ $=39$.

Part III. $(2 \leq i \leq 11, i \neq 3)$. Counting the number of entries of different values in matrix $L_{5}$ gives $c_{2}=38, c_{4}=30, c_{5}=26, c_{6}=22, c_{7}=18, c_{8}=14$, $c_{9}=10, c_{10}=6$, and $c_{11}=38$.

The topological indices, Wiener, hyper Wiener, Harary and Tratch-Stankevitch-Zefirovof, of $B_{n}$ are given in the proposition:

Proposition 3.2. The Wiener, hyper Wiener, Harary, and Tratch-Stankevitch-Zefirov indices of $B_{n}$ are:

1. $W\left(B_{n}\right)=\frac{16}{3} n^{3}+12 n^{2}+\frac{26}{3} n+1$.

2. $W W\left(B_{n}\right)=\frac{8}{3} n^{4}+\frac{32}{3} n^{3}+\frac{46}{3} n^{2}+\frac{37}{3} n+1$

3. $H_{a}\left(B_{n}\right)=4 n+6+(8 n+6)\left(H_{2 n+1}-\frac{11}{6}\right)$, where

$$
H_{2 n+1}=\sum_{l=1}^{2 n+1} \frac{1}{l}
$$

4. $\operatorname{TSZ}\left(B_{n}\right)=\frac{16}{15} n^{5}+\frac{20}{3} n^{4}+16 n^{3}+\frac{55}{3} n^{2}+\frac{254}{15} n+1$

Proof: Proposition can be proven by using Theorem 1 and the relations discussed in the Introduction section.

1. $W\left(B_{n}\right)=\frac{d}{d x}\left[H\left(B_{n}\right)\right]_{x=1}$

$$
\begin{aligned}
& =\frac{d}{d x}\left[(5 n+1) x+(8 n-2) x^{2}+(9 n-6) x^{3}+\sum_{i=4}^{2 n+1}(8 n-4 i+6) x^{i}\right]_{x=1} \\
& =48 n-21+\sum_{i=4}^{2 n+1} i(8 n-4 i+6) \\
& =\frac{16}{3} n^{3}+12 n^{2}+\frac{26}{3} n+1
\end{aligned}
$$

2. $W W\left(B_{n}\right)=\frac{1}{2} \frac{d^{2}}{d x^{2}}\left[x H\left(B_{n}\right)\right]_{x=1}$

$$
\begin{aligned}
& \left.=\frac{1}{2} \frac{d^{2}}{d x^{2}}[5 n+1) x^{2}+(8 n-2) x^{3}+(9 n-6) x^{4}+\sum_{i=4}^{2 n+1}(8 n-4 i+6) x^{i+1}\right]_{x=1} \\
& \left.=83 n-41+\frac{1}{2} \sum_{i=4}^{2 n+1} i(i+1)(8 n-4 i+6)\right] \\
& =\frac{8}{3} n^{4}+\frac{32}{3} n^{3}+\frac{46}{3} n^{2}+\frac{37}{3} n+1
\end{aligned}
$$

3. $H_{a}\left(B_{n}\right)=\int_{0}^{1} \frac{H\left(B_{n}\right)}{x} d x$

$$
\begin{aligned}
& =\int_{0}^{1} \frac{(5 n+1) x+(8 n-2) x^{2}+(9 n-6) x^{3}+\sum_{i=4}^{2 n+1}(8 n-4 i+6) x^{i}}{x} d x \\
& =(5 n+1)+(4 n-1)+(3 n-2)+\sum_{i=4}^{2 n+1} \frac{1}{i}(8 n-4 i+6) \\
& =4 n+6+(8 n+6)\left(H_{2 n+1}-\frac{11}{6}\right) \\
& =\frac{-32}{3} n-5+(8 n+6) H_{2 n+1}
\end{aligned}
$$


4. $\operatorname{TSZ}\left(B_{n}\right)=\frac{1}{3 !} \frac{d^{3}}{d x^{3}}\left[x^{2} H\left(B_{n}\right)\right]_{x=1}$

$=\frac{1}{3 !} \frac{d^{3}}{d x^{3}}\left[(5 n+1) x^{3}+(8 n-2) x^{4}+(9 n-6) x^{5}+\sum_{i=4}^{2 n+1}(8 n-4 i+6) x^{i+2}\right]_{x=1}$

$=\frac{1}{6}\left[6(5 n+1)+24(8 n-2)+60(9 n-6)+\sum_{i=4}^{2 n+1} i(i+1)(i+2)(8 n-4 i+6)\right]$

$=\frac{16}{15} n^{5}+\frac{20}{3} n^{4}+16 n^{3}+\frac{55}{3} n^{2}+\frac{254}{15} n+1$

\section{CONCLUSION}

The aim of this work was to use graph-theoretic tools to predict the physico-chemical properties of organic structures, particularly the linear benzene. A closed form of the Hosoya polynomial was applied to $n$-linear benzene and using the concept of the distance based topological indices, physical, chemical and pharmacological properties of organic molecules can be predicted.

Using the diagrams and distance matrices, the proof of the Hosoya polynomial was done. It was proved that the number of paths of length 1 and 3 are respectively $5 n+1$ and $9 n-6$, and that the number of paths of length $i$, where $2 \leq i \leq 2 n+1$ and $i \neq 3$, is $8 n-4 i+6$. These results were demonstrated with examples. Finally, the Wiener, hyper Wiener, Harary and TSZ indices were obtain to help studying physico-chemical properties of those molecular structures which contain linear benzene as a substructure.

This study opens new research areas for graph theorists, chemists, and pharmacologists.

\section{REFERENCES}

Ali A.A. \& Ahmed M.A. (2011). Hosoya polynomials of pentachains. Communications in Mathematical and in Computer Chemistry 65: 807-819.

Cash G., Klavzar S. \& Petkovsek M. (2002). Three methods for calculation of the hyper-Wiener index of molecular graphs. Journal of Chemical Information and Computer Sciences 42: 571-576.

Diudea M.V. (1996). Wiener and hyper-Wiener numbers in a single matrix. Journal of Chemical Information and Computer Sciences 36: 833-836.

Diudea M.V. (2002). Hosoya polynomial in tori.
Communications in Mathematical and in Computer Chemistry 45: 109-122.

Farahani M.R. (2013). Hosoya, schultz, modified schultz polynomials and their topological indices of benzene molecules: first members of polycyclic aromatic hydrocarbons (PAHs). International Journal of Theoretical Chemistry 1: 09-16.

Gutman I. \& Polansky O.E. (1986). Mathematical Concepts in Organic Chemistry. Springer-Verlag, Berlin, Germany.

Gutman I. (1993). Some properties of the Wiener polynomials. Graph Theory Notes of New York 25: 13-18.

Gutman I., Linert W., Lukovits I. \& Dobrynin A. A. (1997). Trees with extremal hyper-Wiener index: mathematical basis and chemical applications. Journal of Chemical Information and Computer Sciences 37: 349-354.

Hosoya H. (1988). On some counting polynomials in chemistry. Discrete Applied Mathematics 19: 239-257.

Klein D.J., Lukovits I. \& Gutman I. (1995). On the definition of the hyper Wiener index for cycle-containing structures. Journal of Chemical Information and Computer Sciences 35: $50-52$.

Lukovits I. \& Linert W. (1994). A novel definition of the hyperWiener index for cycles. Journal of Chemical Information and Modeling 34: 899-902.

Narayankar Kishori P., Lokesh S.B., Mathad Veena \& Gutman Ivan (2012). Hosoya polynomial of Hanoi graphs. Kragujevac Journal of Mathematics 36: 51-57.

Plavsic D., Nikolic S., Trinajstic N. \& Mihalic Z. (1993). On the Harary index for the characterization of chemical graphs. Journal of Mathematical Chemistry 12: 235-250.

Randic M. (1993). Novel molecular descriptor for structureproperty studies. Chemical Physics Letters 211: 478-483.

Tratch S.S., Stankevitch M.I. \& Zefirov N.S. (1990). Combinatorial models and algorithms in chemistry. the expanded Wiener number - a novel topological index. Journal of Computational Chemistry 11: 899-908. DOI: https://doi.org/10.1002/jcc.540110802

Wiener H. (1947). Structural determination of paraffin boiling points. Journal of the American Chemical Society 69: $17-20$. 